This information is current as of April 25, 2023. 


\title{
Pneumatically Driven Finger Movement: A Novel Passive Functional MR Imaging Technique for Presurgical Motor and Sensory Mapping
}

\author{
S. Shriver, K.E. Knierim, J.P. O'Shea, G.H. Glover, and A.J. Golby
}

\begin{abstract}
SUMMARY: Two of the most common reasons for failure to obtain adequate preoperative functional data are inadequate task performance and excessive head motion. With an MR imaging - compatible pneumatically driven manipulandum, passive motor tasks elicited reproducible contralateral activation in the $\mathrm{Ml}$ and $\mathrm{S} 1$ in 10 healthy controls and 6 patients. The SMA was localized in all healthy controls and in 5 of 6 patients. Head motion was reduced in passive tasks compared with active tasks.
\end{abstract}

ABBREVIATIONS: ANOVA = analysis of variance; $\mathrm{FMRI}=$ functional MR imaging; $\mathrm{BOLD}=$ blood oxygen level-dependent; $\mathrm{MI}=$ primary motor cortex; $\mathrm{SMA}=$ supplementary motor area; $\mathrm{SI}=$ primary somatosensory cortex

$f_{\mathrm{p}}$ MRI of the motor cortex has been shown to be useful in the presurgical evaluation of patients with brain tumors. ${ }^{1}$ However, due to lesion-related neurologic deficits, fMRI may be compromised by the patient's inability to successfully perform relevant tasks. Patients with lesions in and around the sensorimotor strip often have pre-existing weakness or sensory deficits that may hinder their performance during standard fMRI sensorimotor tasks such as finger tapping and hand clenching. Moreover, attempts to compensate for this diminished ability may introduce artifacts (eg, head-motion artifacts due to the recruitment of proximal muscles in patients with cortical weakness). ${ }^{2}$

Any technique used in neurosurgical planning must be able to produce results for single subjects and also to produce activation in motor areas, preferably including the SMA. We present a novel passive-movement task by using a custom-built manipulandum (Mag Design and Engineering, Redwood City, California), which reliably activates the S1, M1, and SMA and reduces overall head motion and task-correlated motion compared with voluntary active-movement paradigms in healthy controls and patients. Pre-

Received January 13, 2011; accepted after revision February 27.

From the Department of Neurosurgery (S.S., K.E.K., J.P.O., A.J.G.), Brigham and Women's Hospital, Harvard Medical School, Boston, Massachusetts; Department of Marketing (S.S.), Fox School of Business, Temple University, Philadelphia, Pennsylvania; and Department of Radiology (G.H.G.), Stanford University School of Medicine, Stanford, California.

This study was supported by grants from the Brain Science Foundation (A.J.G.), Brigham and Women's Institute for the Neurosciences (A.J.G.), NIH KO8 NS048063, U41 RR019703, R25 CA89017 (A.J.G.), and NIH RR P41-09784 (G.H.G.).

Please address correspondence to Alexandra J. Golby, MD, Department of Neurosurgery, Brigham and Women's Hospital, 75 Francis St, Boston, MA 02115; e-mail: agolby@bwh.harvard.edu

-- Indicates open access to non-subscribers at www.ajnr.org

http://dx.doi.org/10.3174/ajnr.A2626 vious research has elicited passive BOLD sensorimotor activation in healthy controls ${ }^{3}$ and sedated pediatric patients. ${ }^{4}$

\section{Technique}

Whole-brain MR imaging was performed on a 3T Excite VH3 MR imaging system with a quadrature head coil (GE Healthcare, Milwaukee, Wisconsin). Participants' heads were immobilized by using soft padding. Conventional structural images and echo-planar functional images were acquired during a 90-minute session.

Ten healthy right-handed volunteers ( 5 women; mean age, 26.9 years) and 6 neurosurgery patients ( 4 women; mean, age 41 years) were recruited. Healthy controls performed a series of 4 passive (right/left index-finger tapping, right/left sequential 5-finger tapping), and 6 active (same as the passive tasks, plus right/left hand clenching) hand-movement tasks while undergoing BOLD echo-planar MR imaging. Four of the 6 patients had preoperative motor deficits, which included hand weakness. Diagnoses included oligoastrocytoma, glioblastoma, and recurrent glioma without anaplastic features. Patients were tested with a clinically relevant subset of the battery with passive and active motor tasks. All tasks consisted of twelve 20-second blocks, alternating between movement and rest. A custom-built MR imaging-compatible pneumatically driven finger-moving device was used for all passive tasks (Mag Design and Engineering). Left- and righthanded devices were attached to the subjects' hands by using Velcro straps (Velcro USA, Manchester, New Hampshire). The devices were controlled with E-Prime stimulus software (Psychology Software Tools, Pittsburgh, Pennsylvania) and were removed during performance of the active tasks.

During the passive tasks, the subjects were instructed to relax while the manipulandum moved their fingers according to the 

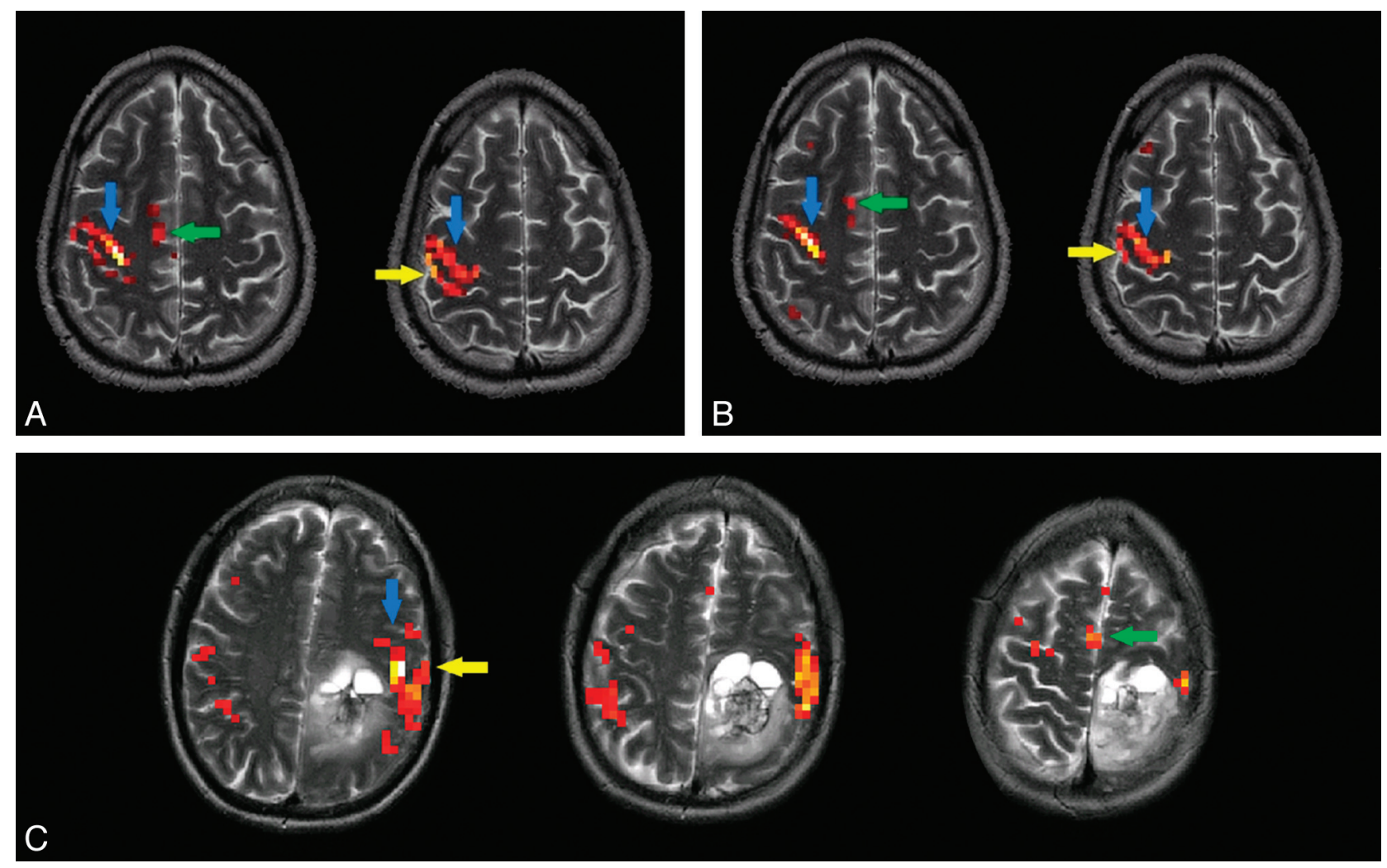

FIG. 1. Activation maps of pneumatically driven left-index-finger movement $(A)$ and volitional left-index-finger movement $(B)$ from a representative healthy control subject; and pneumatically driven sequential right-finger movement $(C)$ from a representative patient with recurrent low-grade glioma and preoperative weakness in the right hand and leg. Tasks display similar activation of S1 (yellow arrows), M1 (blue arrows), and SMA (green arrows). Figures are shown in radiologic convention.

task parameters. For active tasks, verbal instructions were presented through visual display goggles (Resonance Technology, Northridge, California) to perform the task (“GO”) or to rest ("STOP”).

Functional data were reconstructed and corrected for subject motion by using SPM2 (Wellcome Department of Imaging Neuroscience, London, United Kingdom). Spatial smoothing was not performed to preserve the improved spatial resolution of the high-resolution echo-planar images. ${ }^{5}$ Differences between stimulus and rest conditions were examined by using analysis of covariance with global signal-intensity and low-frequency components treated as nuisance covariates. Correction for multiple comparisons was performed by using the theory of Gaussian random fields. ${ }^{6}$ Functional maps were displayed at 3 statistical thresholds: the most stringent, $P<.05$, corrected for multiple comparisons; a less stringent, $P<.001$, uncorrected for multiple comparisons; and the least stringent, $P<.01$, uncorrected.

Head motion was estimated by using the realignment routine in SPM2. The transformation data were then further analyzed by using custom Matlab (MathWorks, Natick, Massachusetts) scripts. For the patients in whom only a subset of tasks was performed, motion parameters were compared between analogous tasks (eg, sequential finger tapping and sequential passive finger movement). Translational and rotational parameters were analyzed separately. The single greatest displacement from each fMRI task was extracted from the SPM2 realignment transformation data by calculating the maximum difference in the absolute values of the realignment parameters between consecutive acquisitions in the time series and analyzed with repeated measures ANOVA with 1 between-subjects factor (controls versus patients) and 1 within-subjects factor (passive versus active). Average motion per TR was also calculated for both translational and rotational movement and subjected to repeated measures ANOVA with 1 between-subjects factor (controls versus patients) and 2 within-subjects factors (task, passive versus active; and direction, $\mathrm{x}, \mathrm{y}, \mathrm{z}$ ). The extent to which head motion was correlated with the stimulus frequency was estimated by calculating the value of the power spectrum of the realignment parameters at the stimulation frequency. ${ }^{7}$ Repeated measures ANOVAs were also performed on the value of the power spectrum.

The data from the passive motor tasks showed contralateral activation in S1 and M1 in all controls and patients. The SMA was activated during the passive tasks in all healthy controls and 5 of 6 patients. fMRI results in healthy controls from the passive tasks were consistent with the analogous active movement tasks with the exception of the passive index finger movement, which tended to have a less robust pattern of activation than the active index finger tapping. Patients' passive tasks also elicited activation comparable with the active tasks (Fig 1). Patients had greater translational head movement than controls (0.44 versus $0.26 \mathrm{~mm}$ ) [F $(1,47)=24.9](P<.0001)$. Passive tasks were associated with less translational movement than active tasks ( 0.34 versus $0.26 \mathrm{~mm}$ ) $[F(1,47)=14.1](P<.001)$. In addition, there was a significant interaction between task and group, so that patients had a greater 
decrease in translational movement for the passive tasks compared with the active tasks than controls $[F(1,47)=5.0](P<$ $.05)$. Rotational-movement passive tasks resulted in less movement compared with active tasks. In patients, however, there was a trend toward decreased rotational movement during the passive tasks. Patients and controls did not differ significantly in rotational movement, and there was not a significant interaction between group and task. Average translational movements per TR were greater in the patients than the controls $[F(1,47)=59.5]$ $(P<.0001)$ and were greater also in the $y$ - and $z$-axes than in the $\mathrm{x}$-axis direction $[F(1,47)=73](P<.0001)$.

\section{DISCUSSION}

Two of the most common reasons for failure to obtain adequate functional data in previous preoperative fMRI studies of patients have been inadequate task performance and excessive head motion. ${ }^{2}$ Our preliminary results by using a performance-invariant passive sensorimotor paradigm using a manipulandum showed reproducible activation of the $\mathrm{S} 1, \mathrm{M} 1$, and SMA in individual healthy controls and patients. The use of the manipulandum reduced head motion compared with active tasks. This reduction was greater for patients than for controls. Task-correlated motion was also decreased, though this was only significant in healthy controls.

Multiple studies have shown a good correspondence between presurgical functional imaging findings and postoperative deficits based on the distance of activation from the lesion boundaries. ${ }^{5,8}$ Many patients with brain tumors or other structural lesions are excluded from functional motor mapping due to their inability to perform relevant tasks. The development of passive tasks can extend the advantages of preoperative fMRI to the subset of neurosurgical candidates with pre-existing deficits. An added advantage of using a passive sensorimotor task is the ability to more precisely control subject performance through standardized tasks, thereby equalizing performance. Standardized tasks rely less on effort or cooperation and thus allow a clearer interpretation of activation maps by decreasing noncontrolled cognitive processes. There is also evidence that the amplitude and frequency of voluntary hand movement influences activation. ${ }^{9-11}$ In sum, use of a manipulan- dum as in this study can decrease confounding factors related to subject compliance and task unpredictability that lead to variability in results.

\section{ACKNOWLEDGMENTS}

The authors thank Julie Levesque for technical support and Ben Krasnow for manipulandum development.

\section{REFERENCES}

1. Schulder M, Maldjian JA, Liu WC, et al. Functional image-guided surgery of intracranial tumors located in or near the sensorimotor cortex. J Neurosurg 1998;89:412-18

2. Krings T, Reinges MH, Erberich S, et al. Functional MRI for presurgical planning: problems, artifacts, and solution strategies. J Neurol Neurosurg Psychiatry 2001;70:749-60

3. Yu N, Estevez N, Hepp-Reymond MC, et al. fMRI assessment of upper extremity related brain activation with an MRI-compatible manipulandum. Int J Comput Assist Radiol Surg 2011;6:447-55. Epub 2010 Aug 10

4. Ogg RJ, Laningham FH, Clarke D, et al. Passive range of motion functional magnetic resonance imaging localizing sensorimotor cortex in sedated children. J Neurosurg Pediatr 2009;4:317-22

5. Yoo SS, Talos IF, Golby AJ, et al. Evaluating requirements for spatial resolution of fMRI for neurosurgical planning. Hum Brain Mapp 2004;21:34-43

6. Friston KJ, Holmes A, Poline JB, et al. Detecting activations in PET and fMRI: levels of inference and power. Neuroimage 1996;4:223-35

7. Hill DL, Smith AD, Simmons A, et al. Sources of error in comparing functional magnetic resonance imaging and invasive electrophysiological recordings. J Neurosurg 2000;93:214-23

8. Morioka T, Shigeto H, Ishibashi H, et al. Magnetic source imaging of the sensory cortex on the surface anatomy MR scanning. Neurol Res 1998;20:235-41

9. Rao SM, Bandettini PA, Binder JR, et al. Relationship between finger movement rate and functional magnetic resonance signal change in human primary motor cortex. J Cereb Blood Flow Metab 1996;16:1250-54

10. Dai TH, Liu JZ, Sahgal V, et al. Relationship between muscle output and functional MRI-measured brain activation. Exp Brain Res 2001;140:290-300

11. Berns GS, Song AW, Mao H. Continuous functional magnetic resonance imaging reveals dynamic nonlinearities of "dose-response" curves for finger opposition. J Neurosci 1999;19:RC17 